\title{
Azelaic Acid
}

National Cancer Institute

\section{Source}

National Cancer Institute. Azelaic Acid. NCI Thesaurus. Code C47407.

A naturally occurring dicarboxylic acid produced by Malassezia furfur and found in whole grain cereals, rye, barley and animal products. Azelaic acid possesses antibacterial, keratolytic, comedolytic, and anti-oxidant activity. Azelaic acid is bactericidal against Proprionibacterium acnes and Staphylococcus epidermidis due to its inhibitory effect on the synthesis of microbial cellular proteins. Azelaic acid exerts its keratolytic and comedolytic effects by reducing the thickness of the stratum corneum and decreasing the number of keratohyalin granules by reducing the amount and distribution of filag grin in epidermal layers. Azelaic acid also possesses a direct anti-inflammatory effect due to its scavenger activity of free oxygen radical. This drug is used topically to reduce inflammation associated with acne and rosacea. 